\title{
Cognitive Medium Access in WLAN Bands: A Real-Time Testbed
}

\author{
(Invited Paper) \\ Stefan Geirhofer, John Z. Sun, and Lang Tong \\ School of Electrical and Computer Engineering \\ Comell University, Ithaca, NY 14853 \\ Email: $\{$ sg355, jzs3,1t35\}@comell.edu
}

\author{
Brian M. Sadler \\ Army Research Laboratory \\ Adelphi, MD 20783-1197 \\ Email: bsadler@arl.army.mil
}

\begin{abstract}
In this paper we present the Cognitive Medium Access Prototype (CMAP), an experimental test bed for dynamic spectrum access in WLAN bands. The prototype builds upon the Cognitive Medium Access (CMA) protocol, and ensures coexistence with the WLAN system by adhering to an interference constraint. CMAP demonstrates the feasibility of CMA's design concept, and validates model assumptions made in proposing this protocol.

CMAP operates in a time-slotted fashion and performs physical-layer sensing and statistical prediction to coexist with the primary user. Its implementation is based on a generalpurpose DSP/FPGA board, which allows for a flexible design. The spectrum sensor; the medium access control, and the cognitive transmitter are discussed in detail. CMAP's performance is evaluated for an experimental coexistence setup, and compared to a blind reference scheme. A significant performance gain, both in terms of increased throughput and reduced interference, is observed.
\end{abstract}

\section{INTRODUCTION}

Wireless communications face an increasing shortage of available frequency bands. This spectrum scarcity has widely been recognized [1], and can be attributed to the inherent inefficiency of statically assigning frequency resources to licensees. With many emerging technologies in this field, such a static allocation concept is approaching its limitations.

Cognitive radio and dynamic spectrum access are emerging research areas that improve spectrum utilization by dynamically accessing spectrum. Researchers have proposed various methodologies for enabling coexistence with license owners. One such approach represents a hierarchical paradigm [2] in which priority is given to primary users, and the cognitive system operates under the constraint that no significant interference is generated.

This interference constraint is enforced by designing the cognitive radio orthogonal to the primary system in either the frequency, space, time, or code domains [3]. In this work we focus on achieving orthogonality in the time domain by transmitting during idle periods between consecutive packet

\footnotetext{
IThis paper was prepared though collaborative participation in the Communications and Networks Consortium sponsored by the U.S. Ammy Research Laboratory under the Collaborative Technology Alliance Program, Cooperative Agreement DAAD19-01-2-0011. The U.S. Government is authorized to reproduce and distribute reprints for Government purposes notwithstanding any copyright notation thereon.
}

transmissions of an IEEE 802.11b WLAN. Interference is limited by constraining the collision rate between both systems.

In designing such a system numerous questions need to be addressed. How accurately can the WLAN's behavior be predicted? How can we quantify the interference that one system inflicts upon the other? Finally, if a packet is dropped, how does the WLAN's retransmission behavior affect the dynamics of the system?

\section{Main contribution}

The Cognitive Medium Access Prototype (CMAP) is an experimental test bed that has been developed with the objective to address the questions raised above. The prototype operates in real-time and performs physical-layer sensing and statistical prediction to avoid collisions with the WLAN. A constraint on the packet error rate is thus enforced. The implementation is described in detail, and CMAP's performance is analyzed for an experimental coexistence setup. Both CMAP's throughput, and the interference it imposes on the WLAN are measured. A significant performance gain -in terms of increased throughput and reduced interference-is realized compared to a blind reference scheme.

\section{B. Related work}

Cognitive Medium Access (CMA) has been proposed as a protocol for improving coexistence with WLAN, based on sensing and statistical prediction. It represents a model-based design, relying on the prediction of white space between packets [4], and using the mathematical framework of constrained Markov decision processes (CMDPs) to obtain the optimal opportunistic medium access control [5]. The prototype CMAP has been developed with the objective of validating model assumptions and demonstrating the feasibility of this approach. Some initial results with CMAP were presented in [6].

A related problem in time domain dynamic spectrum access is considered in [7], where both primary and cognitive system share the same slot structure. Spectrum sensing and medium access are considered, and a separation principle between both problems is derived. For unslotted primaries, a periodic sensing scheme has been introduced in [8].

A test bed for coexistence with multiple WLAN bands has been described in [9]. It is based on the DARPA XG 


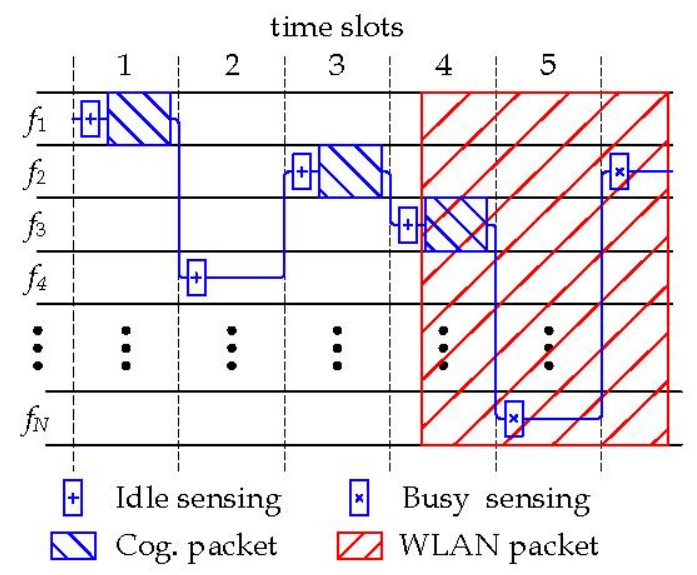

Fig. 1. Physical-layer setup. The frequency hopping, time slotted cognitive radio coexists with an IEEE $802.11 \mathrm{~b}$ WLAN. Sensing is performed at the beginning of every slot. A transmission is initiated based on the outcome of a biased coin flip.

framework an implements a heuristic MAC, which is based on the run-length of observed idle slots.

The setup considered in this paper has conceptual similarities with adaptive frequency hopping, a coexistence mechanism proposed for improving Bluetooth/WLAN coexistence [10]. While there exists a body of literature on the topic, partly developed within the IEEE 802.15.2 task group, our approach is different as it relies on physical-layer sensing and statistical prediction to reduce interference. In a Bluetooth system such sensing is not available, and interference statistics need to be inferred from higher layers.

Organization: The remainder of the paper is organized as follows. In Sec. II the system setup is presented. The implementation of CMAP is discussed in Sec. III, and the coexistence setup in which CMAP's performance is evaluated is presented in Sec. IV. Performance results are shown in Sec. V.

\section{System Setup}

CMA has been developed for a frequency hopping (FH) cognitive radio that coexists with multiple parallel WLAN channels. The protocol performs physical-layer sensing at the beginning of every slot, and transmits only provided that the medium is predicted to be idle for a long enough time.

The CMAP prototype implements this protocol for a single WLAN band due to hardware limitations in the RF front end. Despite this limitation, the prototype is useful for more than demonstrating proof-of-concept. It allows us to measure the dynamic impact that is inflicted upon the WLAN (including retransmissions, etc.), a result that could not easily be obtained otherwise.

In the following an overview of both primary and cognitive system is given.

Primary system: The primary system consists of a single IEEE 802.11b WLAN operating in Channel 6 at $2.437 \mathrm{GHz}$. The system is composed of a router and three PCs with adapter

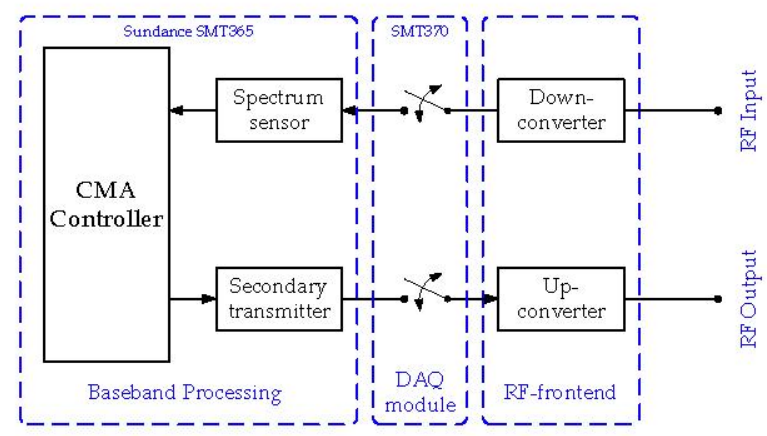

Fig. 2. Block diagram of CMAP.

cards. A packet generator was used to create traffic at different rates (cf. Sec. IV for details).

CMAP Prototype: The cognitive system is a frequency hopping, time slotted system as shown in Fig. 1. At the beginning of every slot, spectrum sensing is performed and if an idle channel is observed, a transmission may be initiated for the remainder of the slot duration based on the outcome of a biased coin flip (cf. Fig. 1). If a busy channel is observed, no transmission is initiated. While the physical-layer sensing ensures that no transmissions take place when the primary system is active, collisions can still occur if the primary user becomes active after sensing has been performed (cf. Fig. 1, slot 4). The transmission probability needs to be carefully designed such that this event occurs infrequent enough to satisfy the interference constraint.

\section{CMAP PROTOTYPE}

In this section the implementation of the prototype CMAP is described. Its operation relies on three main components, namely (i) the spectrum sensor, (ii) the CMA controller, and (iii) the cognitive transmitter. A block diagram of CMAP is shown in Fig. 2. In the following we describe the implementation of these components in detail, followed by the RF front end. Only a single cognitive radio has been developed, but no cognitive receiver.

\section{A. Spectrum sensing}

CMAP performs spectrum sensing at the beginning of every slot in order to determine the state of the channel, i.e., whether the medium is busy or idle. Sensing is based on energydetection, which is mathematically formulated as testing for the hypotheses

$$
\begin{aligned}
& \mathcal{H}_{0}: Y_{i}=N_{i}, 1 \leq i \leq N, \\
& \mathcal{H}_{1}: Y_{i}=S_{i}+N_{i}, 1 \leq i \leq N,
\end{aligned}
$$

where $Y_{i}$ denotes the received signal, $N_{i}$ corresponds to random noise, and $S_{i}$ to the primary user's signal. We assume that $N_{i} \sim \mathcal{C N}\left(0, \sigma_{n}^{2}\right)$ and, given no prior knowledge on the WLAN's transmission behavior, assume $S_{i} \sim \mathcal{C N}\left(0, \sigma_{s}^{2}\right)$.

It is well-known that for this model, a detector based on the Neyman-Pearson criterion corresponds to a threshold on the 
statistic

$$
T\left(y_{1}, \ldots, y_{N}\right)=\sum_{i=1}^{N}\left|y_{i}\right|^{2} \underset{\mathcal{H}_{0}}{\stackrel{\mathcal{H}_{1}}{\gtrless}} \gamma,
$$

where $\gamma$ is chosen such that the probability of false alarm is no greater than some predetermined value. Hence $\gamma$ depends on $\sigma_{s}^{2}$ and $\sigma_{n}^{2}$. In practice, these parameters are unknown and may vary over time. In the implementation, $\gamma$ was chosen heuristically after calibrating the setup.

In the implementation, the following choices were made for the above design parameters. Energy-detection was performed on blocks of $1 \mu \mathrm{s}$, which corresponded to $N=72$ at a sampling rate of $72 \mathrm{MHz}$ and resulted in satisfactory sensing performance. We note that this is a bit shorter than typical sensing times in WLAN. This is due to the high SNR setup, which is of primary concern in this work. If CMAP was used in lower SNR conditions the sensing time may need to be adjusted accordingly.

We note that the sensing requirement is alleviated compared to dynamic spectrum access in the spatial domain [3]. This reflects the fact that in our case orthogonality is ensured by temporal prediction, rather than by spatial separation. An accurate prediction model is crucial to maintain orthogonality.

\section{B. CMA Controller}

It can be shown that, as long as the idle times are approximated by exponential distributions, the optimal control is given by a biased coin flip based on the current sensing result but independent of the sensing history. In the CMA protocol, these transmission probabilities are designed analytically such that the throughput of the cognitive system is maximized subject to interference constraints [5]. In this work, we kept the transmission probability fixed at $p=1 / 3$ and evaluated the performance for varying packet loss. Nevertheless, since both throughput and interference are measured (see Sec. V), the performance of the optimal control can be inferred.

\section{Cognitive transmitter}

CMAP's transmitter is implemented based on a pattern generator which is integrated on the acquisition module, and can be enabled in software. Data contained in an internal buffer is then transferred to the DAC and played back in an infinite loop. This functionality was used to generate a narrowband FH signal. The signal did not hop within the band but always occupied a $1 \mathrm{MHz}$ wide band aligned with the center frequency of the WLAN band. This leads to a "strict" collision model in which every packet collision results in a packet drop. This is a worst-case assumption that is frequently made in such coexistence setups [11].

Modulation parameters for the cognitive transmitter were chosen similar to the Bluetooth standard [12]. Gaussian Frequency Shift Keying (GFSK) with a time-bandwidth product of 0.3 was used at a symbol-rate of $1 \mathrm{MSymbols} / \mathrm{s}$. The slot length of the system was chosen as $T_{s}=625 \mu \mathrm{s}$.

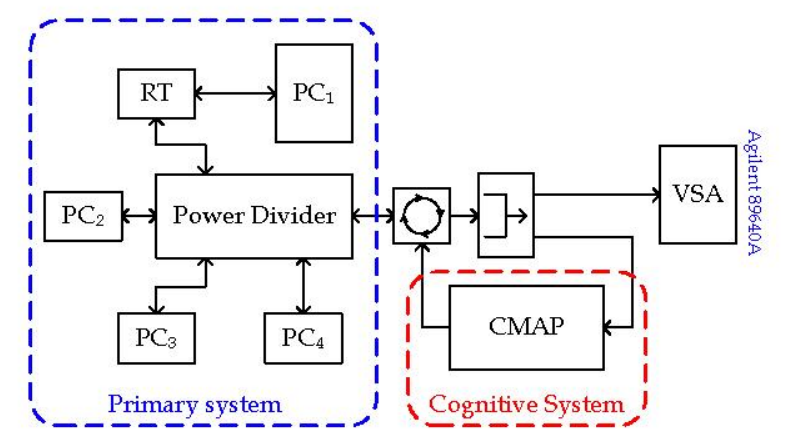

Fig. 3. Measurement setup used for performance assessment. The WLAN system and CMAP are connected via a resistive power diver, leading to an $\mathrm{RF}$ isolated setup.

\section{RF front end}

An RF front end is used to down- and up-convert the base band signals to the center frequency of $2.437 \mathrm{GHz}$. Down-conversion was performed using an evaluation board of a commercial WLAN chipset. It performed direct downconversion, and integrated a low noise amplifier (LNA) as well as intermediate filters. The board could be controlled via proprietary software to optimize signal levels. The upconversion was performed using an Agilent E4438C vector signal generator.

\section{Measurement Setup}

The previous section described the implementation of CMAP. Due to the hierarchical configuration, in which the cognitive radio performs sensing and statistical prediction to improve coexistence, its performance depends on the primary user's activity. Therefore, the coexistence setup in which CMAP operates forms a crucial part of any performance comparison.

The interaction between both systems is characterized by physical-layer parameters such as propagation, power- and interference levels, as well as the primary's traffic scenario. The focus of this work is to examine CMAP's performance with respect to varying traffic load. To this end we define a metric, which reflects the "busyness" of the WLAN. In order to focus on the prediction performance, we configure the power levels such that every collision between both systems inevitably results in a packet drop. This is a worst-case scenario in practice [5].

\section{A. Hardware setup}

The coexistence setup used in our performance analysis is depicted in Fig. 3 and consists of the WLAN system (the primary user), the prototype CMAP, and monitoring tools that validate the operation of the system.

The primary system consists of a commercial WLAN router and three workstations with WLAN adapter cards. All devices operate in the same channel according to the IEEE 802.11b standard. The router is connected to another workstation via ethernet. This computer can transmit and receive data, 
and additionally runs packet capturing software to count the number of successfully received packets.

The RF connection between primaries is made by connecting all devices to a resistive power divider using coaxial cables. While this does not necessarily reflect the propagation characteristics encountered in practice it enables us to obtain simple and invariant propagation conditions. Additionally, this setup guarantees repeatable measurement results and ensures that the setup does not incur unrelated interference from adjacent WLAN devices (the measurements were taken in an office building).

The prototype CMAP is connected to the same power divider. Its input thus consists of the superposition of the WLAN signals (just as it would if the devices transmitted using antennas). CMAP's output is fed back to the system via a circulator:

The power levels of the WLAN devices and CMAP are adjusted such that every packet collision inevitably causes a packet drop. The collision model was verified experimentally, by having CMAP transmit deterministically in every second slot. In this case, the time between cognitive transmissions is smaller than the packet duration and hence none of the WLAN packets can be transmitted without a collision. We verified by experiment that in this case the WLAN throughput reduces to zero, as needed.

\section{B. Traffo characteristics}

The mutual interference, generated by a packet collision, is determined by the physical layer model. The collision rate itself, however, depends on the primary user's traffic characteristics, and CMAP's ability to avoid collisions by sensing and statistical prediction. In accordance with other papers in the coexistence literature, we measure the throughput of both primary and cognitive system with respect to the offered traffic load of the WLAN. The term "offered" emphasizes that this traffic load may not be achievable by the physical layer, even in the case of no interference. In order to provide for a more intuitive comparison we normalize the traffic load (in average packets per second) by the maximum achievable rate of the WLAN, and denote this normalized rate as $\sigma$. The case $\sigma=1$ thus corresponds to the maximum rate supported by the physical layer. We are primarily interested in WLAN traffic loads $0 \leq \sigma \leq 1$, as this corresponds to achievable loads in the interference-free case.

The analysis focuses on the average throughput that can be achieved for both systems for stationary traffic scenarios. In particular, we consider constant-payload UDP traffic with exponentially distributed inter-arrival times at different rates. The "D-ITG" traffic generator [13] was used to generate the traffic. Further details on the traffic generation can be found in [14].

While performance is evaluated within a stationary traffic scenario (at different loads $\sigma$ ), we expect our results to extend to instationary traffic, as well. Provided that model parameters are tracked over time, CMA can be adapted such as to maintain interference constraints [4]

\section{Measurement methodology}

The measurements were performed in the following manner. For each WLAN traffic load $\sigma$, packets were generated the corresponding rate. These packets were captured at the workstation comected to the WLAN router using a commercial software tool. Based on these traces the average throughput of the WLAN was calculated.

After establishing the traffic scenario, CMAP was enabled which typically resulted in some decrease of the WLAN's throughput, due to residual collisions. The WLAN's throughput was again obtained, now with CMAP enabled. At the same time, CMAP also counted the number of initiated and successful transmissions, which was in turn used to calculate the throughput of the cognitive system.

For each of the measured traffic loads $\sigma$, we obtained the throughput for CMAP, a blind reference scheme, and the interference-free case to provide proper comparison.

In this context we define the term throughput as the average number of successfully transmitted packets, both for the WLAN and CMAP, respectively. For the WLAN, a packet transmission is successful if it is correctly received by the router. For the CMAP, we define a transmission to be successful if the medium remains idle throughout the entire slot period in which the transmission takes place. In the measurement setup this is reported if a packet transmission is initiated and the medium is observed idle at the begiming of the next slot period. This criterion seems appropriate since no cognitive transmitter/receiver pair is available and WLAN packets are always longer than the slot period.

\section{MEAsUREMENT REsUlTs}

The performance of CMAP is shown in Fig. 4 with respect to the traffic load $\sigma$. The throughput of the cognitive system is shown in Fig. 4(a) and the impact on the WLAN is shown in Fig 4(b). Specifically, Fig. 4(b) shows the fraction of successfully received packets compared to the total traffic load. Hence, in the interference-free case we would expect this metric to be unity across all $\sigma$. The measurement results indeed confirm this expectation with good accuracy.

The performance of CMAP is compared to a blind reference scheme, which does not perform sensing but deterministically transmits in every tenth slot. CMAP outperforms the blind reference scheme both in tems of throughput and interference. In the following we address throughput and interference plots separately, but stress that both were obtained from the same measurement and are hence directly related. Clearly, it is possible to trade off cognitive throughput for reduced interference.

CMAP outperforms the blind reference in terms of throughput. For low $\sigma$, the throughput converges to the transmission rates of both systems. At $\sigma=0$, the primary system is inactive: the blind reference transmits in every tenth slot, and CMAP in every third idle slot. The measurement reflects this behavior as can be seen in Fig. 4. The throughput of both systems decreases with $\sigma$ but CMAP outperforms the blind transmitter significantly up to $\sigma \approx 0.5$, at which point both schemes saturate. For large $\sigma$, we observe a small residual throughput, 


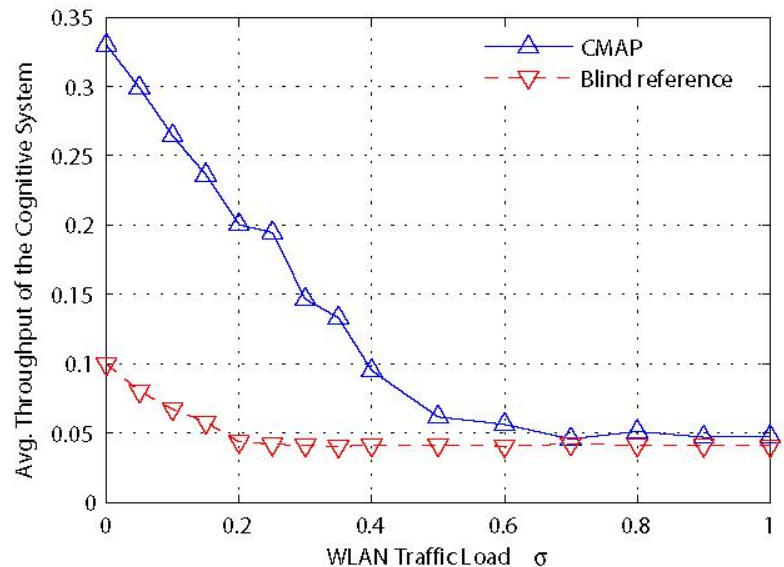

(a) Cognitive System

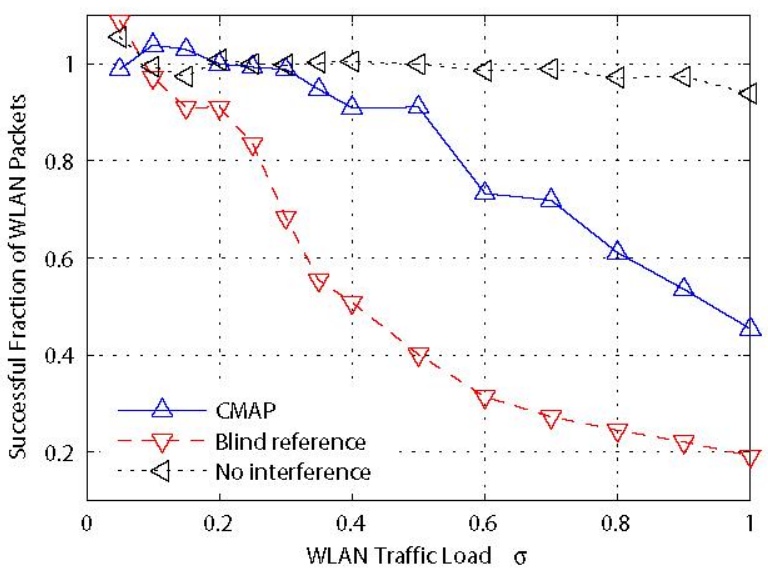

(b) WLAN System

Fig. 4. Measurement result. CMAP outperforms a blind reference scheme both in terms of throughput and interference. The throughput of the cognitive system (average number of successful packet transmissions per unit time) is shown in (a), its impact on the WLAN in (b). Fig. (b) plots the successfully received fraction of transmitted WLAN packets. The fraction was obtained by dividing the observed number of packets by the mean transmit rate; hence this metric may exceed one for finite measurement duration.

which is due to backoff periods of the WLAN that are present even at high traffic load.

The interference, which CMAP inflicts upon the WLAN, is quantified in Fig. 4 again with respect to the traffic load $\sigma$. We observe that CMAP outperforms the blind reference in terms of interference as well. For low $\sigma$ the WLAN remains almost unaffected by the presence of the cognitive system. As $\sigma$ is increased the effects become more pronounced, both for CMAP and the blind reference. This can be attributed to the WLAN's retransmission behavior. At low $\sigma$, the channel is predominantly idle and packet retransmissions can be accommodated. At high $\sigma$, however, this becomes impossible as the medium is too busy to support the required number of retransmissions.

Throughput and interference are directly related to the transmission probability of the cognitive system. For instance, by reducing the transmission probability, interference can be reduced at the expense of cognitive throughput. In this way, by adapting the transmission probability according to traffic load, the cognitive system can adhere to an interference constraint.

In summary, the measurement shows that CMAP significantly outperforms the blind transmitter and that thus a significant performance gain is associated with introducing sensing and cooperation in this framework.

\section{CONCLUSion}

In conclusion we have presented CMAP, a real-time test bed for improving coexistence with WLAN channels. The prototype is fundamentally based on physical-layer sensing and prediction of the WLAN's medium access, and has conceptual similarities with a Bluetooth system. CMAP operates in real-time and hence allows us to assess the cognitive radio's dynamic impact on an actual WLAN system.

\section{REFERENCES}

[1] Proceedings of the First IEEE International Symposium on New Frontiers in Dynamic Spectrum Access Networks, Nov. 2005.

[2] Q. Zhao and B. M. Sadler, "Dynamic Spectrum Access: Signal Processing, Networking, and Regulatory Policy," IEEE Signal Processing Mag., vol. 55 , no. 5, pp. 2294-2309, May 2007.

[3] I. Akyildiz, W. Lee, M. Vuran, and S. Mohanty, "NeXt generation/dynamic spectrum access/cognitive radio wireless networks: A survey," Computer Networks, vol. 50, no. 13, pp. 2127-2159, Sept. 2006.

[4] S. Geirhofer, L. Tong, and B. M. Sadler, "Dynamic Spectrum Access in the Time Domain: Modeling and Exploiting Whitespace," IEEE Commun. Mag., vol. 45, no. 5, pp. 66-72, May 2007.

[5] — "Cognitive Medium Access: Constraining Interference Based on Experimental Models," to appear in IEEE Journal on Selected Areas in Communications, Jan. 2008.

[6] S. Geirhofer, J. Z. Sun, L. Tong, and B. M. Sadler, "CMAP: A RealTime Prototype for Cognitive Medium Access," in Proc. IEEE Military Communications Conference (MILCM), Oct. 2007.

[7] Q. Zhao, L. Tong, A. Swami, and Y. Chen, "Decentralized Cognitive MAC for Opportunistic Spectrum Access in Ad Hoc Networks: A POMDP Framework," IEEE J. Select. Areas Commun, vol. 25, no. 3 , pp. 589-600, Apr. 2007.

[8] Q. Zhao, S. Geirhofer, L. Tong, and B. M. Sadler, "Opportunistic Spectrum Access via Periodic Channel Sensing," to appear in IEEE Trans. Sig. Proc., Feb. 2008.

[9] S. D. Jones, N. Merheb, and I.-J. Wang, "An experiment for sensingbased opportunistic spectrum access in CSMA/CA networks," in First IEEE Internatial Symposium on New Frontiers in Dynamic Spectrum Access Networks, Nov. 2005, pp. 593-596.

[10] C.-F. Chiasserini and R. R. Rao, "Coexistence Mechanisms for Interference Mitigation in the 2.4-GHz ISM Band," IEEE Trans. Wireless Commun., vol. 2, no. 5, pp. 964-975, Sept. 2003.

[11] Golmie, N. and Van Dyck, R. E. and Soltanian, A. and Tonnerre, A. and Rébala, O., "Interference Evaluation of Bluetooth and IEEE 802.11b Systems," Wireless Networks, vol. 9, no. 3, pp. 201-211, May 2003.

[12] Bluetooth Special Interest Group, "Specification of the Bluetooth System," Nov. 2004.

[13] S. Avallone, A. Botta, D. Emma, S. Guadagno, and A. Pescape, "DITG V.2.4 Manual," University of Napoli "Federio II", Tech. Rep., Dec. 2004.

[14] S. Geirhofer, L. Tong, and B. M. Sadler, "Dynamic Spectrum Access in WLAN Channels: Empirical Model and Its Stochastic Analysis," in Proc. First International Workshop on Technology and Policy for Accessing Spectrum, 2006. 